\title{
Sign changes of error terms related to arithmetical functions
}

\author{
par PAUlo J. ALMEIDA
}

\begin{abstract}
RÉSUmÉ. Soit $H(x)=\sum_{n \leq x} \frac{\phi(n)}{n}-\frac{6}{\pi^{2}} x$. Motivé par une conjecture de Erdös, Lau a développé une nouvelle méthode et il a démontré que $\#\{n \leq T: H(n) H(n+1)<0\} \gg T$. Nous considérons des fonctions arithmétiques $f(n)=\sum_{d \mid n} \frac{b_{d}}{d}$ dont l'addition peut être exprimée comme $\sum_{n \leq x} f(n)=\alpha x+P(\log (x))+E(x)$. Ici $P(x)$ est un polynôme, $E(x)=-\sum_{n \leq y(x)} \frac{b_{n}}{n} \psi\left(\frac{x}{n}\right)+o(1)$ avec $\psi(x)=x-\lfloor x\rfloor-1 / 2$. Nous généralisons la méthode de Lau et démontrons des résultats sur le nombre de changements de signe pour ces termes d'erreur.

AbStract. Let $H(x)=\sum_{n \leq x} \frac{\phi(n)}{n}-\frac{6}{\pi^{2}} x$. Motivated by a conjecture of Erdös, Lau developed a new method and proved that $\#\{n \leq T: H(n) H(n+1)<0\} \gg T$. We consider arithmetical functions $f(n)=\sum_{d \mid n} \frac{b_{d}}{d}$ whose summation can be expressed as $\sum_{n \leq x} f(n)=\alpha x+P(\log (x))+E(x)$, where $P(x)$ is a polynomial, $E(x)=-\sum_{n \leq y(x)} \frac{b_{n}}{n} \psi\left(\frac{x}{n}\right)+o(1)$ and $\psi(x)=x-\lfloor x\rfloor-1 / 2$. We generalize Lau's method and prove results about the number of sign changes for these error terms.
\end{abstract}

\section{Introduction}

We say that an arithmetical function $f(x)$ has a sign change on integers at $x=n$, if $f(n) f(n+1)<0$. The number of sign changes on integers of $f(x)$ on the interval $[1, T]$ is defined as

$$
N_{f}(T)=\#\{n \leq T, n \text { integer }: f(n) f(n+1)<0\} .
$$

We also define $z_{f}(T)=\#\{n \leq T, n$ integer : $f(n)=0\}$. Throughout this work, $\psi(x)=x-\lfloor x\rfloor-1 / 2$ and $f(n)$ will be an arithmetical function such

Manuscrit reçu le 8 janvier 2006.

This work was funded by Fundação para a Ciência e a Tecnologia grant number $\mathrm{SFRH} / \mathrm{BD} / 4691 / 2001$, support from my advisor and from the department of mathematics of University of Georgia. 
that

$$
f(n)=\sum_{d \mid n} \frac{b_{d}}{d} \text { for some sequence of real numbers } b_{n} .
$$

The motivation for our work was a paper by Y.-K. Lau [5], where he proves that the error term, $H(x)$, given by

$$
\sum_{n \leq x} \frac{\phi(n)}{n}=\frac{6}{\pi^{2}} x+H(x)
$$

has a positive proportion of sign changes on integers solving a conjecture stated by P. Erdös in 1967.

An important tool that Lau used to prove his theorem, was that the error term $H(x)$ can be expressed as

(1) $\quad H(x)=-\sum_{n \leq \frac{x}{\log ^{5} x}} \frac{\mu(n)}{n} \psi\left(\frac{x}{n}\right)+O\left(\frac{1}{\log ^{20} x}\right), \quad$ (S. Chowla [3])

We generalize Lau's result in the following way

Theorem 1.1. Suppose $H(x)$ is a function that can be expressed as

$$
H(x)=-\sum_{n \leq y(x)} \frac{b_{n}}{n} \psi\left(\frac{x}{n}\right)+O\left(\frac{1}{k(x)}\right),
$$

where each $b_{n}$ is a real number and

(i) $y(x)$ increasing, $x^{\frac{1}{4}} \ll y(x) \ll \frac{x}{(\log x)^{5+\frac{D}{2}}}$, for some $D>0$, and

$$
\sum_{n \leq x} b_{n}^{4} \ll x \log ^{D} x
$$

(ii) $k(x)$ is an increasing function, satisfying $\lim _{x \rightarrow \infty} k(x)=\infty$.

(iii) $H(x)=H(\lfloor x\rfloor)-\alpha\{x\}+\theta(x)$, where $\alpha \neq 0$ and $\theta(x)=o(1)$.

Let $\prec \in\{<,=, \leq\}$. If $\#\{1 \leq n \leq T: \alpha H(n) \prec 0\} \gg T$ then there exists a positive constant $c_{0}$ and $c_{0} T$ disjoint subintervals of $[1, T]$, with each of them having at least two integers, $m$ and $n$, such that $\alpha H(m)>0$ and $\alpha H(n) \prec 0$. In particular,

(1) $\#\{n \leq T: \alpha H(n)>0\} \gg T$;

(2) if $\#\{n \leq T: \alpha H(n)<0\} \gg T$, then $N_{H}(T) \gg T$ or $z_{H}(T) \gg T$.

We consider arithmetical functions $f(n)$ for which, the error term of the summation function satisfies the conditions of Theorem 1.1. A first class is described in the following result 
Theorem 1.2. Let $f(n)$ be an arithmetical function and suppose the sequence $b_{n}$ satisfies condition (3) and

$$
\sum_{n \leq x} b_{n}=B x+O\left(\frac{x}{\log ^{A} x}\right)
$$

for some $B$ real, $D>0$ and $A>6+\frac{D}{2}$, respectively. Let $\alpha=\sum_{n=1}^{\infty} \frac{b_{n}}{n^{2}}$,

$\gamma_{b}=\lim _{x \rightarrow \infty}\left(\sum_{n \leq x} \frac{b_{n}}{n}-B \log x\right)$ and $H(x)=\sum_{n \leq x} f(n)-\alpha x+\frac{B \log 2 \pi x}{2}+\frac{\gamma_{b}}{2}$.

If $\alpha \neq 0$, then Theorem 1.1 is valid for the error term $H(x)$. Moreover, if $f(n)$ is a rational function, then, except when $\alpha=0$, or $B=0$ and $\alpha$ is rational, we have

$$
N_{H}(T) \gg T \quad \text { if and only if } \quad \#\{n \leq T: \alpha H(n)<0\} \gg T .
$$

Notice that this class of arithmetical functions is closed for addition, i.e., if $f(n)$ and $g(n)$ are members of the class then also is $(f+g)(n)$. In the case considered by Lau, it was known that $H(x)$ has a positive proportion of negative values (Y.-F. S. Pétermann [6]), so the second part of Theorem 1.2 generalizes Lau's result. Another example is $f(n)=\frac{n}{\phi(n)}$.

Using a result of U. Balakrishnan and Y.-F. S. Pétermann [2] we are able to apply Theorem 1.1 to more general arithmetical functions:

Theorem 1.3. Let $f(n)$ be an arithmetical function and suppose the sequence $b_{n}$ satisfies condition (3) and

$$
\sum_{n=1}^{\infty} \frac{b_{n}}{n^{s}}=\zeta^{\beta}(s) g(s)
$$

for some $\beta$ real, $D>0$, and a function $g(s)$ with a Dirichlet series expansion absolutely convergent for $\sigma>1-\lambda$, for some $\lambda>0$. Let $\alpha=\zeta^{\beta}(2) g(2)$ and

$$
H(x)= \begin{cases}\sum_{n \leq x} f(n)-\alpha x, & \text { if } \beta<0, \\ \sum_{n \leq x} f(n)-\alpha x-\sum_{j=0}^{\lfloor\beta} B_{j}(\log x)^{\beta-j} & \text { if } \beta>0,\end{cases}
$$

where the constants $B_{j}$ are well defined. If $\alpha \neq 0$, then Theorem 1.1 is valid for the error term $H(x)$.

Theorem 1.3 is valid for the following examples, where $r \neq 0$ is real:

$$
\left(\frac{\phi(n)}{n}\right)^{r}, \quad\left(\frac{\sigma(n)}{n}\right)^{r}, \quad\left(\frac{\phi(n)}{\sigma(n)}\right)^{r} .
$$




\section{Main Lemma}

The main tool used by Y.-K. Lau was his Main Lemma, where he proved that if $H(x)=\sum_{n \leq x} \frac{\phi(n)}{n}-\frac{6}{\pi^{2}} x$ then

$$
\int_{T}^{2 T}\left(\int_{t}^{t+h} H(u) \mathrm{d} u\right)^{2} \mathrm{~d} t \ll T h
$$

for sufficiently large $T$ and any $1 \leq h \ll \log ^{4} T$. Lau's argument depends essentially on the formula (1). In this section, we obtain a generalization of Lau's Main Lemma.

Main Lemma. Suppose $H(x)$ is a function that can be expressed as (2) and satisfies conditions (i) and (ii) of Theorem 1.1. Then, for all large $T$ and $h \leq \min \left(\log T, k^{2}(T)\right)$, we have

$$
\int_{T}^{2 T}\left(\int_{t}^{t+h} H(u) \mathrm{d} u\right)^{2} \mathrm{~d} t \ll T h^{\frac{3}{2}} .
$$

For any positive integer $N$, define

$$
H_{N}(x)=-\sum_{d \leq N} \frac{b_{d}}{d} \psi\left(\frac{x}{d}\right) .
$$

The Main Lemma will follow from the next result.

Lemma 2.1. Assume the conditions of the Main Lemma and take $D>0$ satisfying condition (i). Let $E=4+\frac{D}{2}$, then

(a) For any $\delta>0$, large $T$, any $Y \ll T$ and $N \leq y(T)$, we have

$$
\int_{T}^{T+Y}\left(H(u)-H_{N}(u)\right)^{2} \mathrm{~d} u \ll \frac{Y}{N^{1-\delta}}+\frac{Y}{k^{2}(T)}+y(T+Y)(\log T)^{E} ;
$$

(b) For all large $T, N \leq y(T)$ and $1 \leq h \leq \min \left(\log T, k^{2}(T)\right)$, we have

$$
\int_{T}^{2 T}\left(\int_{t}^{t+h} H_{N}(u) \mathrm{d} u\right)^{2} \mathrm{~d} t \ll T h^{\frac{3}{2}}+N^{3}(\log N)^{E} .
$$

Now we prove the Main Lemma:

Proof. Take $N=T^{\frac{1}{4}}$ and $\delta>0$ small. Cauchy's inequality gives us

$$
\begin{aligned}
\left(\int_{t}^{t+h} H(u) \mathrm{d} u\right)^{2} \leq 2 & \left(\int_{t}^{t+h} H_{N}(u) \mathrm{d} u\right)^{2} \\
& +2\left(\int_{t}^{t+h}\left(H(u)-H_{N}(u)\right) \mathrm{d} u\right)^{2} .
\end{aligned}
$$


Since $N=T^{\frac{1}{4}}$ then, for sufficiently large $T, N^{3} \log ^{E} N \ll T$. So, using part (b) of Lemma 2.1 we have

$$
\int_{T}^{2 T}\left(\int_{t}^{t+h} H_{N}(u) \mathrm{d} u\right)^{2} \mathrm{~d} t \ll T h^{\frac{3}{2}}+N^{3} \log ^{E} N \ll T h^{\frac{3}{2}} .
$$

Using Cauchy's inequality and interchanging the integrals,

$$
\begin{aligned}
\int_{T}^{2 T} & \left(\int_{t}^{t+h}\left(H(u)-H_{N}(u)\right) \mathrm{d} u\right)^{2} \mathrm{~d} t \\
& \leq h \int_{T}^{2 T}\left(\int_{t}^{t+h}\left(H(u)-H_{N}(u)\right)^{2} \mathrm{~d} u\right) \mathrm{d} t \\
& \leq h \int_{T}^{2 T+h}\left(\int_{\max (u-h, T)}^{\min (u, 2 T)}\left(H(u)-H_{N}(u)\right)^{2} \mathrm{~d} t\right) \mathrm{d} u \\
& \leq h^{2} \int_{T}^{2 T+h}\left(H(u)-H_{N}(u)\right)^{2} \mathrm{~d} u \\
& \ll h^{2}\left(\frac{T+h}{N^{1-\delta}}+\frac{T+h}{k^{2}(T)}+y(2 T+h)(\log T)^{E}\right) \\
& \ll T+T h \ll T h^{\frac{3}{2}}
\end{aligned}
$$

since $y(2 T+h) \ll \frac{T}{(\log T)^{E+1}}$ and $h \leq \min \left(\log T, k^{2}(T)\right)$. Hence

$$
\int_{T}^{2 T}\left(\int_{t}^{t+h} H(u) \mathrm{d} u\right)^{2} \mathrm{~d} t \ll T h^{\frac{3}{2}} .
$$

\section{Step I}

In this section, we will prove part (a) of Lemma 2.1. Using expression (2) and Cauchy's inequality, we obtain

$$
\int_{T}^{T+Y}\left(H(u)-H_{N}(u)\right)^{2} \mathrm{~d} u \leq 2 \int_{T}^{T+Y}\left(\sum_{m=N+1}^{y(u)} \frac{b_{m}}{m} \psi\left(\frac{u}{m}\right)\right)^{2} \mathrm{~d} u+O\left(\frac{Y}{k^{2}(T)}\right) .
$$

Let $\eta(T, m, n)=\max \left(T, y^{-1}(m), y^{-1}(n)\right)$, then

$$
2 \int_{T}^{T+Y}\left(\sum_{m=N+1}^{y(u)} \frac{b_{m}}{m} \psi\left(\frac{u}{m}\right)\right)^{2} \mathrm{~d} u=2 \sum_{m, n=N+1}^{y(T+Y)} \frac{b_{m} b_{n}}{m n} \int_{\eta(T, m, n)}^{T+Y} \psi\left(\frac{u}{m}\right) \psi\left(\frac{u}{n}\right) \mathrm{d} u .
$$


The Fourier series of $\psi(u)=u-\lfloor u\rfloor-\frac{1}{2}$, when $u$ is not an integer, is given by

$$
\psi(u)=-\frac{1}{\pi} \sum_{k=1}^{\infty} \frac{\sin (2 \pi k u)}{k}
$$

so we obtain

$$
\frac{2}{\pi^{2}} \sum_{m, n=N+1}^{y(T+Y)} \frac{b_{m} b_{n}}{m n} \sum_{k, l=1}^{\infty} \frac{1}{k l} \int_{\eta(T, m, n)}^{T+Y} \sin \left(2 \pi \frac{k u}{m}\right) \sin \left(2 \pi \frac{l u}{n}\right) \mathrm{d} u .
$$

Now, the integral above is equal to

$$
\frac{1}{2} \int_{\eta(T, m, n)}^{T+Y} \cos \left(2 \pi u\left(\frac{k}{m}+\frac{l}{n}\right)\right)-\cos \left(2 \pi u\left(\frac{k}{m}-\frac{l}{n}\right)\right) \mathrm{d} u .
$$

For the first term we get

$$
\int_{\eta(T, m, n)}^{T+Y} \cos \left(2 \pi u\left(\frac{k}{m}+\frac{l}{n}\right)\right) \mathrm{d} u \ll \frac{1}{\left(\frac{k}{m}+\frac{l}{n}\right)} .
$$

If $\frac{k}{m}=\frac{l}{n}$, then

$$
\int_{\eta(T, m, n)}^{T+Y} \cos \left(2 \pi u\left(\frac{k}{m}-\frac{l}{n}\right)\right) \mathrm{d} u \leq Y
$$

otherwise

$$
\int_{\eta(T, m, n)}^{T+Y} \cos \left(2 \pi u\left(\frac{k}{m}-\frac{l}{n}\right)\right) \mathrm{d} u \ll \frac{1}{\left|\frac{k}{m}-\frac{l}{n}\right|} .
$$

Part (a) of Lemma 2.1 will now follow from the next three lemmas.

Lemma 3.1. Let $E=4+\frac{D}{2}$ as in Lemma 2.1. Then

$$
\sum_{m, n \leq X}\left|b_{m} b_{n}\right| \sum_{\substack{k, l=1 \\ k n \neq l m}}^{\infty} \frac{1}{k l|k n-l m|} \ll X(\log X)^{E} .
$$

Lemma 3.2. If $D>0$ satisfies condition (3), then

$$
\sum_{m, n \leq X}\left|b_{m} b_{n}\right| \sum_{k, l=1}^{\infty} \frac{1}{k l(k n+l m)} \ll X(\log X)^{1+\frac{D}{2}} .
$$

Lemma 3.3. For any $\delta>0$,

$$
\sum_{N<m, n \leq X} \frac{\left|b_{m} b_{n}\right|}{m n} \sum_{\substack{k, l=1 \\ k n=l m}}^{\infty} \frac{1}{k l} \ll \frac{1}{N^{1-\delta}} .
$$


In order to finish the proof of part (a) of Lemma 2.1 we just need to take $X=y(T+Y)$ in the previous lemmas. Hence

$$
\int_{T}^{T+Y}\left(H(u)-H_{N}(u)\right)^{2} \mathrm{~d} u \ll \frac{Y}{N^{1-\delta}}+y(T+Y)(\log T)^{E}+\frac{Y}{k^{2}(T)} .
$$

Before we prove the three lemmas above, we need the following technical result

Lemma 3.4. Let $b_{n}$ be a sequence satisfying condition (3). Then

$$
\begin{aligned}
\sum_{n \leq N} b_{n}^{2} \ll N \log ^{\frac{D}{2}} N, \quad \sum_{n \leq N}\left|b_{n}\right| \ll N \log ^{\frac{D}{4}} N, & \sum_{n \leq N} \frac{b_{n}^{2}}{n} \ll(\log N)^{1+\frac{D}{2}}, \\
\sum_{n \leq N} \frac{\left|b_{n}\right|}{n} \ll(\log N)^{1+\frac{D}{4}}, \quad \sum_{n>N} \frac{b_{n}^{4}}{n^{2}} \tau(n) \ll \frac{1}{N^{1-\delta}}, & \text { for any } \delta>0 .
\end{aligned}
$$

Proof. Follows from Cauchy's inequality, partial summations and the fact that, for any $\epsilon>0, \tau(n)=O\left(n^{\epsilon}\right)$.

Remark. If $H(x)$ can be expressed in the form (2), then

$$
|H(x)| \leq \sum_{n \leq y(x)} \frac{\left|b_{n}\right|}{n}+O\left(\frac{1}{k(x)}\right) \ll(\log x)^{1+\frac{D}{4}} .
$$

Proof of Lemma 3.2 : Since the arithmetical mean is greater or equal to the geometrical mean, we have

$$
\begin{aligned}
\sum_{m, n \leq X}\left|b_{m} b_{n}\right| \sum_{k, l=1}^{\infty} \frac{1}{k l(k n+l m)} & \leq 2 \sum_{m, n \leq X}\left|b_{m} b_{n}\right| \sum_{k, l=1}^{\infty} \frac{1}{k l \sqrt{k n l m}} \\
& \ll\left(\sum_{m \leq X} \frac{\left|b_{m}\right|}{\sqrt{m}}\right)^{2} \\
& \ll\left(\sum_{m \leq X} 1\right)\left(\sum_{M \leq X} \frac{b_{M}^{2}}{M}\right) \\
& \ll X(\log X)^{1+\frac{D}{2}} .
\end{aligned}
$$

Proof of Lemma 3.3 : For the second sum, take $d=(m, n), m=d \alpha$ and $n=d \beta$. Since $k n=l m$, then $\alpha \mid k$ and $\beta \mid l$. Taking $k=\alpha \gamma$, we also have $l=\beta \gamma$. As

$$
\sum_{\substack{k, l=1 \\ k n=l m}}^{\infty} \frac{1}{k l}=\frac{1}{\alpha \beta} \sum_{\gamma=1}^{\infty} \frac{1}{\gamma^{2}}=\frac{\pi^{2}}{6} \frac{(m, n)^{2}}{m n}
$$


Then,

$$
\begin{aligned}
\sum_{N<m, n \leq X} \frac{\left|b_{m} b_{n}\right|}{m n} \sum_{\substack{k, l=1 \\
k n=l m}}^{\infty} \frac{1}{k l} & =\frac{\pi^{2}}{6} \sum_{\substack{N<m, n \leq X \\
m^{2} n^{2}}} \frac{\left|b_{m} b_{n}\right|(m, n)^{2}}{m^{2}} \\
& \leq \frac{\pi^{2}}{6} \sum_{d \leq X}\left(d \sum_{\substack{N<m \leq X \\
d \mid m}} \frac{\left|b_{m}\right|}{m^{2}}\right)^{2} .
\end{aligned}
$$

The next step is to estimate the inner sum using Hölder inequality

$$
\left(\sum_{\substack{N<m \leq X \\ d \mid m}} \frac{\left|b_{m}\right|}{m^{2}}\right)^{2} \leq\left(\sum_{\substack{N<m \leq X \\ d \mid m}} \frac{b_{m}^{4}}{m^{2}}\right)^{\frac{1}{2}}\left(\sum_{\substack{N<M \leq X \\ d \mid M}} \frac{1}{M^{2}}\right)^{\frac{3}{2}}
$$

Set $M=\beta d$, then

$$
\left(\sum_{\substack{N<M \leq X \\ d \mid M}} \frac{1}{M^{2}}\right)^{\frac{3}{2}}=\frac{1}{d^{3}}\left(\sum_{\frac{N}{d}<\beta \leq \frac{X}{d}} \frac{1}{\beta^{2}}\right)^{\frac{3}{2}} \ll \frac{1}{d^{3}}\left(\min \left\{1, \frac{d^{3}}{N^{3}}\right\}\right)^{\frac{1}{2}}
$$

To complete the proof of Lemma 3.3 we use Cauchy's inequality and Lemma 3.4. For any $\delta>0$,

$$
\sum_{N<m, n \leq X} \frac{\left|b_{m} b_{n}\right|}{m n} \sum_{\substack{k, l=1 \\ k n=l m}}^{\infty} \frac{1}{k l} \ll \sum_{d \leq X}\left(\frac{1}{d}\left(\min \left\{1, \frac{d^{3}}{N^{3}}\right\}\right)^{\frac{1}{2}}\left(\sum_{\substack{N<m \leq X \\ d \mid m}} \frac{b_{m}^{4}}{m^{2}}\right)^{\frac{1}{2}}\right)
$$

therefore

$$
\begin{aligned}
\left(\sum_{N<m, n \leq X} \frac{\left|b_{m} b_{n}\right|}{m n} \sum_{\substack{k, l=1 \\
k n=l m}}^{\infty} \frac{1}{k l}\right)^{2} & \ll \sum_{d \leq X} \frac{1}{d^{2}} \min \left\{1, \frac{d^{3}}{N^{3}}\right\} \sum_{D \leq X}\left(\sum_{\substack{N<m \leq X \\
D \mid m}} \frac{b_{m}^{4}}{m^{2}}\right) \\
& \ll\left(\frac{1}{N^{3}} \sum_{d \leq N} d+\sum_{d>N} \frac{1}{d^{2}}\right) \sum_{N<m \leq X}\left(\frac{b_{m}^{4}}{m^{2}} \sum_{D \mid m} 1\right) \\
& \ll \frac{1}{N} \sum_{N<m \leq X} \frac{b_{m}^{4}}{m^{2}} \tau(m) \ll \frac{1}{N^{2-\delta}} .
\end{aligned}
$$

Proof of Lemma 3.1 : This lemma is a generalization of Hilfssatz 6 in [9] of A. Walfisz. Notice first that

$$
\sum_{m, n \leq X}\left(\left|b_{m} b_{n}\right| \sum_{\substack{k, l=1 \\ k n \neq l m}}^{\infty} \frac{1}{k l|k n-l m|}\right) \leq 2 \sum_{m \leq n \leq X}\left(\left|b_{m} b_{n}\right| \sum_{\substack{k, l=1 \\ k n \neq l m}}^{\infty} \frac{1}{k l|k n-l m|}\right) .
$$


Like in [9] we begin by separating the interior sum into four terms:

$$
\begin{aligned}
\sum_{\substack{k, l=1 \\
k n \neq l m}}^{\infty} \frac{1}{k l|k n-l m|} & =\sum_{\substack{k, l=1 \\
l m \leq \frac{k n}{2}}}^{\infty}\left(\frac{1}{k l|k n-l m|}\right)+\sum_{\substack{k, l=1 \\
\frac{k n}{2}<l m<k n}}^{\infty}\left(\frac{1}{k l|k n-l m|}\right) \\
& +\sum_{\substack{k, l=1 \\
k n<l m<2 k n}}^{\infty}\left(\frac{1}{k l|k n-l m|}\right)+\sum_{\substack{k, l=1 \\
l m \geq 2 k n}}^{\infty}\left(\frac{1}{k l|k n-l m|}\right)
\end{aligned}
$$

For the first term, we use Lemma 3.4,

$$
\begin{aligned}
\sum_{m \leq n \leq X}\left|b_{m} b_{n}\right| \sum_{\substack{k, l=1 \\
l m \leq \frac{k n}{2}}}^{\infty} \frac{1}{k l|k n-l m|} & \leq 2 \sum_{m \leq n \leq X}\left|b_{m} b_{n}\right| \sum_{\substack{k, l=1 \\
l m \leq \frac{k n}{2}}}^{\infty} \frac{1}{k^{2} l n} \\
& =2 \sum_{m \leq n \leq X}\left|b_{m}\right| \frac{\left|b_{n}\right|}{n} \sum_{k=1}^{\infty} \frac{1}{k^{2}} \sum_{l \leq \frac{k n}{2 m}} \frac{1}{l} \\
& \ll \sum_{m \leq n \leq X}\left|b_{m}\right| \frac{\left|b_{n}\right|}{n} \sum_{k=1}^{\infty}\left(\frac{\log k}{k^{2}}+\frac{\log X}{k^{2}}\right) \\
& \ll \log X \sum_{n \leq X} \frac{\left|b_{n}\right|}{n} \sum_{m \leq n}\left|b_{m}\right| \ll X(\log X)^{1+\frac{D}{2}}
\end{aligned}
$$

From the forth inequality of Lemma 3.4, we get

$$
\sum_{m \leq n \leq X}\left|b_{m} b_{n}\right| \sum_{\substack{k, l=1 \\ k n \leq \frac{l m}{2}}}^{\infty} \frac{1}{k l|k n-l m|} \ll X(\log X)^{1+\frac{D}{2}}
$$

The estimation of the third term is more complicated and we have to use a different approach. In this case, $\frac{1}{l}<\frac{2 m}{k n}$, so that

$$
\begin{aligned}
\sum_{m \leq n \leq X}\left|b_{m} b_{n}\right| \sum_{\substack{k, l=1 \\
\frac{k n}{2}<l m<k n}}^{\infty} \frac{1}{k l|k n-l m|} & \\
& <2 \sum_{m \leq n \leq X}\left|b_{m}\right| \frac{\left|b_{n}\right|}{n} \sum_{k=1}^{\infty} \frac{1}{k^{2}} \sum_{\frac{k n}{2 m}<l<\frac{k n}{m}} \frac{m}{k n-l m}
\end{aligned}
$$




$$
\begin{aligned}
<2 \sum_{m \leq n \leq X}\left|b_{m}\right| \frac{\left|b_{n}\right|}{n} & \sum_{k=1}^{\infty} \frac{1}{k^{2}} \sum_{l \leq \frac{k n}{m}-1}\left(\frac{1}{\frac{k n}{m}-l}\right) \\
& +2 \sum_{m \leq n \leq X}\left|b_{m}\right| \frac{\left|b_{n}\right|}{n} \sum_{k=1}^{\infty} \frac{1}{k^{2}} \sum_{\frac{k n}{m}-1<l<\frac{k n}{m}} \frac{m}{k n-l m} .
\end{aligned}
$$

Now, taking $L=\left[\frac{k n}{m}-l\right]$,

$$
\begin{aligned}
\sum_{m \leq n \leq X}\left|b_{m}\right| \frac{\left|b_{n}\right|}{n} \sum_{k=1}^{\infty} \frac{1}{k^{2}} \sum_{l \leq \frac{k n}{m}-1}\left(\frac{1}{\frac{k n}{m}-l}\right) & \leq \sum_{m \leq n \leq X}\left|b_{m}\right| \frac{\left|b_{n}\right|}{n} \sum_{k=1}^{\infty} \frac{1}{k^{2}} \sum_{L \leq \frac{k n}{m}-1} \frac{1}{L} \\
& \ll X(\log X)^{1+\frac{D}{2}}
\end{aligned}
$$

as in the first term. If there exists an integer $l$ with $\frac{k n}{m}-1<l<\frac{k n}{m}$, then $m \nmid k n$. In this case, $k n-l m=m\left\{\frac{k n}{m}\right\}$ and $m<n$. So, we have to estimate

$$
\sum_{m<n \leq X}\left|b_{m}\right| \frac{\left|b_{n}\right|}{n} \sum_{\substack{k=1 \\ m \nmid k n}}^{\infty} \frac{1}{k^{2}\left\{\frac{k n}{m}\right\}}
$$

Notice that the fractional part of $\frac{k n}{m}$ is at least $\frac{1}{m}$. So, when $k \geq m$,

$$
\sum_{m<n \leq X}\left|b_{m}\right| \frac{\left|b_{n}\right|}{n} \sum_{k=m}^{\infty} \frac{m}{k^{2}} \ll \sum_{m<n \leq X}\left|b_{m}\right| \frac{\left|b_{n}\right|}{n} \ll X(\log X)^{\frac{D}{2}} .
$$

We are left with the estimation of

$$
\sum_{m<n \leq X}\left|b_{m}\right| \frac{\left|b_{n}\right|}{n} \sum_{\substack{k<m \\ m \nmid k n}} \frac{1}{k^{2}\left\{\frac{k n}{m}\right\}} .
$$

Since $m \nmid k n$, given $k$ and $n$, we can take $a_{k, n}$, such that $1 \leq a_{k, n}<m$ and $a_{k, n} \equiv k n \bmod m$. Then,

$$
\begin{aligned}
\sum_{m<n \leq X}\left|b_{m}\right| \frac{\left|b_{n}\right|}{n} \sum_{\substack{k<m \\
m \nmid k n}} \frac{1}{k^{2}\left\{\frac{k n}{m}\right\}} & \leq \sum_{m<n \leq X}\left|b_{m}\right| \frac{\left|b_{n}\right|}{n} \sum_{k<m} \frac{m}{k^{2} a_{k, n}} \\
& \leq \sum_{a, k \leq X} \frac{1}{a k^{2}} \sum_{\max (a, k)<m \leq X} m\left|b_{m}\right| \sum_{\substack{m<n \leq X \\
k n \equiv a \\
\bmod m}} \frac{\left|b_{n}\right|}{n} .
\end{aligned}
$$

We need to estimate the inner sums. In order to do that, we partition the interval $[1, X]$ in intervals of the form $[M, 2 M)$ and apply Cauchy's 
inequality. Take $1 \leq P \leq Q \leq X$, then,

$$
\sum_{P \leq m<2 P} m\left|b_{m}\right| \sum_{\substack{Q \leq n<2 Q \\ k n \equiv a \bmod m}} \frac{\left|b_{n}\right|}{n} \ll \frac{P}{Q} \sum_{P \leq m<2 P}\left|b_{m}\right| \sum_{\substack{Q \leq n<2 Q \\ k n \equiv a \bmod m}}\left|b_{n}\right| .
$$

Next, we apply Cauchy's inequality twice, first to the first sum on the right and afterwards to the second sum:

$$
\begin{aligned}
& \left(\sum_{P \leq m<2 P}\left(\left|b_{m}\right| \sum_{\substack{Q \leq n<2 Q \\
k n \equiv a \bmod m}}\left|b_{n}\right|\right)\right)^{2} \\
& \leq \sum_{P \leq M<2 P} b_{M}^{2} \sum_{P \leq m<2 P}\left(\sum_{\substack{Q \leq n<2 Q \\
k n \equiv a \bmod m}}\left|b_{n}\right|\right)^{2} \\
& \ll P \log ^{\frac{D}{2}} P \sum_{P \leq m<2 P}\left(\sum_{\substack{Q \leq n<2 Q \\
k n \equiv a}} b_{n}^{2} \sum_{\substack{Q \leq N<2 Q \\
k N \equiv a}} 1\right) \\
& \ll P \bmod m \\
& \sum_{\substack{Q \leq \frac{D}{2} \\
P \leq m<2 P}}\left(\left(1+\frac{Q}{\frac{m}{(k, m)}}\right) \sum_{\substack{Q \leq n<2 Q \\
k n \equiv a \bmod m}} b_{n}^{2}\right) .
\end{aligned}
$$

Since $m \leq 2 P \leq 2 Q$, we have $\frac{Q}{m} \geq \frac{1}{2}$. Using also $(k, m) \leq k$, we obtain

$$
1+\frac{Q}{\frac{m}{(k, m)}} \leq 1+\frac{Q k}{m} \leq \frac{Q}{m}(k+2) \leq 3 \frac{Q k}{m} .
$$

Therefore,

$$
\begin{aligned}
& \left(\sum_{P \leq m<2 P}\left(\left|b_{m}\right| \sum_{\substack{Q \leq n<2 Q \\
k n \equiv a \bmod m}}\left|b_{n}\right|\right)\right)^{2} \ll P \log ^{\frac{D}{2}} P \sum_{P \leq m<2 P}\left(3 \frac{Q k}{m} \sum_{\substack{Q \leq n<2 Q \\
k n \equiv a \bmod m}} b_{n}^{2}\right) \\
& \ll P \frac{3 k Q}{P} \log ^{\frac{D}{2}} P \sum_{Q \leq n<2 Q}\left(b_{n}^{2} \sum_{\substack{P \leq m<2 P \\
m \mid k n-a}} 1\right) \\
& \ll k Q(\log P)^{\frac{D}{2}} \sum_{Q \leq n<2 Q} b_{n}^{2} \tau(k n-a) .
\end{aligned}
$$


By a theorem of S. Ramanujan [7], $\sum_{n \leq X} \tau^{2}(n) \sim X \log ^{3} X$ and by another application of Cauchy inequality and condition (3), we get

$$
\begin{aligned}
\left(\sum_{Q \leq n<2 Q} b_{n}^{2} \tau(k n-a)\right)^{2} & \leq\left(\sum_{Q \leq n<2 Q} b_{n}^{4}\right)\left(\sum_{Q \leq n<2 Q} \tau^{2}(k n-a)\right) \\
& \ll Q \log ^{D} Q \sum_{k Q-a \leq N<2 k Q-a} \tau^{2}(N) \\
& \ll k Q^{2} \log ^{D+3} X .
\end{aligned}
$$

Therefore,

$$
\begin{aligned}
\sum_{P \leq m<2 P}\left(m\left|b_{m}\right| \sum_{\substack{Q \leq n<2 Q \\
k n \equiv a \bmod m}} \frac{\left|b_{n}\right|}{n}\right) & \ll \frac{P}{Q}\left(k Q(\log P)^{\frac{D}{2}} \sum_{Q \leq n<2 Q} b_{n}^{2} \tau(k n-a)\right)^{\frac{1}{2}} \\
& \ll \frac{P}{Q}\left(k Q(\log X)^{\frac{D}{2}}\left(k Q^{2} \log ^{D+3} X\right)^{\frac{1}{2}}\right)^{\frac{1}{2}} \\
& \ll P k^{\frac{3}{4}}(\log X)^{1+\frac{D}{2}} .
\end{aligned}
$$

The number of pairs of intervals of the form $([P, 2 P),[Q, 2 Q))$ to be considered is at most $\ll \log ^{2} X$, hence

$$
\begin{aligned}
\sum_{a, k \leq X} \frac{1}{a k^{2}} \sum_{a<m \leq X} m\left|b_{m}\right| \sum_{\substack{m<n \leq X \\
k n \equiv a \bmod m}} \frac{\left|b_{n}\right|}{n} & \ll \sum_{a, k \leq X} \frac{k^{\frac{3}{4}}}{a k^{2}} \sum_{P, Q} P(\log X)^{1+\frac{D}{2}} \\
& \ll X(\log X)^{4+\frac{D}{2}}
\end{aligned}
$$

The fourth term is treated as the third, hence

$$
\sum_{m \leq n \leq X}\left|b_{m} b_{n}\right| \sum_{\substack{k, l=1 \\ k n<l m<2 k n}}^{\infty} \frac{1}{k l|k n-l m|} \ll X(\log X)^{4+\frac{D}{2}} .
$$

This completes the proof of Lemma 3.1.

\section{Step II}

In this section we prove part (b) of Lemma 2.1. From equation (8), we get

$$
\int_{a}^{b} \psi(u) \mathrm{d} u=\frac{1}{2 \pi^{2}} \sum_{k=1}^{\infty}\left(\frac{\cos (2 \pi k b)}{k^{2}}-\frac{\cos (2 \pi k a)}{k^{2}}\right) .
$$


Using the definition of $H_{N}$ stated in (7), we obtain

$$
\begin{aligned}
\int_{t}^{t+h} H_{N}(u) \mathrm{d} u & =-\sum_{m \leq N} \frac{b_{m}}{m} \int_{t}^{t+h} \psi\left(\frac{u}{m}\right) \mathrm{d} u \\
& =-\frac{1}{2 \pi^{2}} \sum_{m \leq N} b_{m} \sum_{k=1}^{\infty} \frac{\cos \left(2 \pi \frac{k(t+h)}{m}\right)-\cos \left(2 \pi \frac{k t}{m}\right)}{k^{2}}
\end{aligned}
$$

As usual, let us write $e(t)$ for $e^{2 \pi i t}$, then

$$
\int_{t}^{t+h} H_{N}(u) \mathrm{d} u=\frac{1}{4 \pi^{2}} \sum_{m \leq N} b_{m} \sum_{k=1}^{\infty} \frac{\left(e\left(\frac{k h}{m}\right)-1\right) e\left(\frac{k t}{m}\right)\left(e\left(-k \frac{(2 t+h)}{m}\right)-1\right)}{k^{2}} .
$$

Therefore,

$$
\begin{aligned}
& 16 \pi^{4} \int_{T}^{2 T}\left|\int_{t}^{t+h} H_{N}(u) \mathrm{d} u\right|^{2} \mathrm{~d} t \\
& =\int_{T}^{2 T}\left|\sum_{m \leq N} b_{m} \sum_{k=1}^{\infty} \frac{\left(e\left(\frac{k h}{m}\right)-1\right) e\left(\frac{k t}{m}\right)\left(e\left(-k \frac{(2 t+h)}{m}\right)-1\right)}{k^{2}}\right|^{2} \mathrm{~d} t \\
& =\sum_{m, n \leq N} b_{m} b_{n} \sum_{k, l=1}^{\infty} \frac{\left(e\left(\frac{k h}{m}\right)-1\right)\left(e\left(-\frac{l h}{n}\right)-1\right)}{(k l)^{2}} \\
& \times \int_{T}^{2 T} e\left(\frac{k t}{m}\right) e\left(-\frac{l t}{n}\right)\left(e\left(-k \frac{(2 t+h)}{m}\right)-1\right)\left(e\left(l \frac{(2 t+h)}{n}\right)-1\right) \mathrm{d} t .
\end{aligned}
$$

After multiplying the terms inside the integral above, we obtain the following four terms that we will estimate below:

$$
\begin{aligned}
& \int_{T}^{2 T} e\left(\frac{k t}{m}-\frac{l t}{n}\right) \mathrm{d} t+e\left(\frac{l h}{n}-\frac{k h}{m}\right) \int_{T}^{2 T} e\left(\frac{l t}{n}-\frac{k t}{m}\right) \mathrm{d} t \\
& -e\left(-\frac{k h}{m}\right) \int_{T}^{2 T} e\left(-\frac{l t}{n}-\frac{k t}{m}\right) \mathrm{d} t-e\left(\frac{l h}{n}\right) \int_{T}^{2 T} e\left(\frac{l t}{n}+\frac{k t}{m}\right) \mathrm{d} t .
\end{aligned}
$$


Notice that, $\left|\int_{T}^{2 T} e^{2 \pi i r t} \mathrm{~d} t\right| \leq \frac{1}{\pi|r|}$, for any $r \neq 0$. We begin with the last term and use $\mid e(t)-1) \mid \leq 2$ and Lemma 3.2. Then

$$
\begin{aligned}
& \left|\sum_{m, n \leq N} b_{m} b_{n} \sum_{k, l=1}^{\infty} \frac{\left(e\left(\frac{k h}{m}\right)-1\right)\left(e\left(-\frac{l h}{n}\right)-1\right) e\left(\frac{l h}{n}\right)}{(k l)^{2}} \int_{T}^{2 T} e\left(\frac{l t}{n}+\frac{k t}{m}\right) \mathrm{d} t\right| \\
& \leq \frac{4}{\pi} \sum_{m, n \leq N}\left|b_{m} b_{n}\right| \sum_{k, l=1}^{\infty} \frac{1}{(k l)^{2}\left(\frac{l}{n}+\frac{k}{m}\right)} \\
& \ll \sum_{m, n \leq N}\left|b_{m} b_{n}\right| \sum_{k, l=1}^{\infty}\left(\frac{m}{k}\right)\left(\frac{n}{l}\right) \frac{1}{k l(l m+k n)} \ll N^{3}(\log N)^{1+\frac{D}{2}} .
\end{aligned}
$$

The third term is treated similarly to obtain

$$
\begin{array}{r}
\left|\sum_{m, n \leq N} b_{m} b_{n} \sum_{k, l=1}^{\infty} \frac{\left(e\left(\frac{k h}{m}\right)-1\right)\left(e\left(-\frac{l h}{n}\right)-1\right) e\left(-\frac{k h}{m}\right)}{(k l)^{2}} \int_{T}^{2 T} e\left(-\frac{l t}{n}-\frac{k t}{m}\right) \mathrm{d} t\right| \\
\ll N^{3}(\log N)^{1+\frac{D}{2}} .
\end{array}
$$

Now, if $k n=l m$, then

$$
\int_{T}^{2 T} e\left(\frac{k t}{m}-\frac{l t}{n}\right) \mathrm{d} t+e\left(\frac{l h}{n}-\frac{k h}{m}\right) \int_{T}^{2 T} e\left(\frac{l t}{n}-\frac{k t}{m}\right) \mathrm{d} t=2 T .
$$

If $k n \neq l m$ then,

$$
\int_{T}^{2 T} e\left(\frac{k t}{m}-\frac{l t}{n}\right) \mathrm{d} t+e\left(\frac{l h}{n}-\frac{k h}{m}\right) \int_{T}^{2 T} e\left(\frac{l t}{n}-\frac{k t}{m}\right) \mathrm{d} t \ll \frac{1}{\left|\frac{k}{m}-\frac{l}{n}\right|} .
$$

Let us study first the case when $k n \neq l m$,

$$
\begin{aligned}
& \left|\sum_{m, n \leq N} b_{m} b_{n} \sum_{\substack{k, l=1 \\
k n \neq l m}}^{\infty} \frac{\left(e\left(\frac{k h}{m}\right)-1\right)\left(e\left(-\frac{l h}{n}\right)-1\right)}{(k l)^{2}} \int_{T}^{2 T} e\left(\frac{k t}{m}-\frac{l t}{n}\right) \mathrm{d} t\right| \\
& \ll \sum_{m, n \leq N}\left|b_{m} b_{n}\right| \sum_{\substack{k, l=1 \\
k n \neq l m}}^{\infty} \frac{1}{(k l)^{2}\left|\frac{k}{m}-\frac{l}{n}\right|} \\
& \ll \sum_{m, n \leq N}\left|b_{m} b_{n}\right| \sum_{\substack{k, l=1 \\
k n \neq l m}}^{\infty}\left(\frac{m}{k}\right)\left(\frac{n}{l}\right) \frac{1}{k l|k n-l m|} \ll N^{3} \log ^{E} N,
\end{aligned}
$$


by Lemma 3.1. Similarly,

$$
\begin{gathered}
\left|\sum_{m, n \leq N} b_{m} b_{n} \sum_{\substack{k, l=1 \\
k n \neq l m}}^{\infty} \frac{\left(e\left(\frac{k h}{m}\right)-1\right)\left(e\left(-\frac{l h}{n}\right)-1\right) e\left(\frac{l h}{n}-\frac{k h}{m}\right)}{(k l)^{2}} \int_{T}^{2 T} e\left(\frac{l t}{n}-\frac{k t}{m}\right) \mathrm{d} t\right| \\
\ll N^{3} \log ^{E} N .
\end{gathered}
$$

If $k n=m l$, we will use $|e(t)-1| \ll \min (1,|t|)$ instead. The expression obtained has some similarities with Lemma 3.3. We are going to use the same argument to prove:

$$
\sum_{m, n \leq N}\left|b_{m} b_{n}\right| \sum_{\substack{k, l=1 \\ k n=l m}}^{\infty} \frac{1}{(k l)^{2}} \min \left(1, \frac{k h}{m}\right) \min \left(1, \frac{l h}{n}\right) \ll h^{\frac{3}{2}} .
$$

As in Lemma 3.3, take $d=(m, n), \alpha=\frac{m}{d}, \beta=\frac{n}{d}$ and $\gamma=\frac{k}{\alpha}$. So $l=\beta \gamma$ and then

$$
\sum_{\substack{k, l=1 \\ k n=l m}}^{\infty} \frac{1}{(k l)^{2}} \min \left(1, \frac{k h}{m}\right) \min \left(1, \frac{l h}{n}\right)=\frac{1}{\alpha^{2} \beta^{2}} \sum_{\gamma=1}^{\infty} \frac{1}{\gamma^{4}}\left(\min \left(1, \frac{h \gamma}{d}\right)\right)^{2} .
$$

If $d \leq h$, we obtain $\sum_{\gamma=1}^{\infty} \frac{1}{\gamma^{4}}\left(\min \left(1, \frac{h \gamma}{d}\right)\right)^{2}=\frac{\pi^{4}}{90}$, and if $h<d \leq N$,

$$
\begin{aligned}
\sum_{\gamma=1}^{\infty} \frac{1}{\gamma^{4}}\left(\min \left(1, \frac{h \gamma}{d}\right)\right)^{2} & =\left(\frac{h}{d}\right)^{2} \sum_{\gamma \leq \frac{d}{h}} \frac{1}{\gamma^{2}}+\sum_{\gamma>\frac{d}{h}} \frac{1}{\gamma^{4}} \\
& \ll\left(\frac{h}{d}\right)^{2}+\left(\frac{h}{d}\right)^{3} \ll\left(\frac{h}{d}\right)^{2} .
\end{aligned}
$$

Therefore,

$$
\begin{aligned}
& \sum_{m, n \leq N}\left|b_{m} b_{n}\right| \sum_{\substack{k, l=1 \\
k n=l m}}^{\infty} \frac{1}{(k l)^{2}} \min \left(1, \frac{k h}{m}\right) \min \left(1, \frac{l h}{n}\right) \\
& =\sum_{m, n \leq N}\left|b_{m} b_{n}\right| \frac{(m, n)^{4}}{m^{2} n^{2}} \sum_{\gamma=1}^{\infty} \frac{1}{\gamma^{4}}\left(\min \left(1, \frac{h \gamma}{(m, n)}\right)\right)^{2} \\
& \leq \sum_{d \leq N} d^{4} \sum_{\substack{m, n \leq N \\
d=(m, n)}} \frac{\left|b_{m} b_{n}\right|}{m^{2} n^{2}} \sum_{\gamma=1}^{\infty} \frac{1}{\gamma^{4}}\left[\min \left(1, \frac{h \gamma}{d}\right)\right]^{2} \\
& \ll \sum_{d \leq h}\left(d^{2} \sum_{\substack{m \leq N \\
d \mid m}} \frac{\left|b_{m}\right|}{m^{2}}\right)^{2}+h^{2} \sum_{h<d \leq N}\left(d \sum_{\substack{m \leq N \\
d \mid m}} \frac{\left|b_{m}\right|}{m^{2}}\right)^{2} .
\end{aligned}
$$


Since $d \mid m$ we have $m>h$ and so, $h^{2} \sum_{h<d \leq N}\left(d \sum_{\substack{m \leq N \\ d \mid m}} \frac{\left|b_{m}\right|}{m^{2}}\right)^{2} \ll h^{1+\delta}$. To estimate the first term we begin with Hölder inequality:

$$
\sum_{d \leq h} d^{4}\left(\sum_{\substack{m \leq N \\ d \mid m}} \frac{\left|b_{m}\right|}{m^{2}}\right)^{2} \leq \sum_{d \leq h} d^{4}\left(\sum_{\substack{m \leq N \\ d \mid m}} \frac{\left|b_{m}\right|^{4}}{m^{2}}\right)^{\frac{1}{2}}\left(\sum_{\substack{M \leq N \\ d \mid M}} \frac{1}{M^{2}}\right)^{\frac{3}{2}} .
$$

The third sum is $O\left(\frac{1}{d^{3}}\right)$ (similar to (11)). Then

$$
\begin{aligned}
\sum_{d \leq h} d^{4}\left(\sum_{\substack{m \leq N \\
d \mid m}} \frac{\left|b_{m}\right|^{4}}{m^{2}}\right)^{\frac{1}{2}}\left(\sum_{\substack{M \leq N \\
d \mid M}} \frac{1}{M^{2}}\right)^{\frac{3}{2}} & \ll \sum_{d \leq h} d\left(\sum_{\substack{d \leq m \leq N \\
d \mid m}} \frac{\left|b_{m}\right|^{4}}{m^{2}}\right)^{\frac{1}{2}} \\
& \ll\left(\sum_{d \leq h} d^{2}\right)^{\frac{1}{2}}\left[\sum_{D \leq h}\left(\sum_{\substack{D \leq m \leq N \\
D \mid m}} \frac{\left|b_{m}\right|^{4}}{m^{2}}\right)\right]^{\frac{1}{2}} \\
& \ll h^{\frac{3}{2}}\left(\sum_{m \leq N} \frac{\left|b_{m}\right|^{4}}{m^{2}} \sum_{\substack{D \leq h \\
D \mid m}} 1\right)^{\frac{1}{2}} \\
& \ll h^{\frac{3}{2}}\left(\sum_{m \leq N} \frac{\left|b_{m}\right|^{4}}{m^{2}} \tau(m)\right)^{\frac{1}{2}} .
\end{aligned}
$$

The last part of Lemma 3.4 implies $\sum_{m<N} \frac{\left|b_{m}\right|^{4}}{m^{2}} \tau(m)=O(1)$, where the underlying constant doesn't depend on $N$. Therefore, we obtain inequality (13) and part (b) of Lemma 2.1, follows.

\section{A general Theorem}

In this section, we prove Theorem 1.1, from which the main Theorems 1.2 and 1.3 , will be deduced.

Proof of Theorem 1.1 : From the Main Lemma, we have, for all large $T$ and $h \leq \min \left(\log T, k^{2}(T)\right)$,

$$
\int_{T}^{2 T}\left(\int_{t}^{t+h} H(u) \mathrm{d} u\right)^{2} \mathrm{~d} t \ll T h^{\frac{3}{2}}
$$

Assume $\#\{n \leq T: \alpha H(n) \prec 0\} \gg T$. Let $c>0$ be a constant and $T$ be sufficiently large, such that $\#\{n \leq 2 T: \alpha H(n) \prec 0\}>c T$. Divide the interval $[1,2 T]$ into subintervals of length $h$, where $h$ is a sufficiently large integer satisfying $h \leq \log T$. Then more than $c T / h$ of those subintervals must have at least one integer $n$ with $\alpha H(n) \prec 0$. Let $\mathcal{C}$ be the set of the subintervals which satisfy this property. Write $\mathcal{C}=\left\{J_{r} \mid 1 \leq r \leq R\right\}$, where the subintervals are indexed by their positions in the interval $[1,2 T]$ and where $R>c T / h$. Define $K_{s}=J_{3 s-2}$, for $1 \leq s \leq R / 3$, and let $\mathcal{D}$ be the 
set of these subintervals. We have $\#(\mathcal{D})>c T / 3 h$. Notice that any two members of $\mathcal{D}$ are separated by a distance of at least $2 h$.

Let $M$ be the number of subintervals $K$ in $\mathcal{D}$ for which there exists an integer $n$ in $K$ such that $\alpha H(n) \prec 0$ and $\alpha H(m) \leq 0$ for every integer $m \in(n, n+2 h)$, and let $\mathcal{S}$ be the set of the corresponding values of $n$.

Lemma 5.1. For some absolute constant $c_{1}$, we have $M \leq c_{1} \frac{T}{h^{\frac{3}{2}}}$.

Proof. Since $H(x)=H(\lfloor x\rfloor)-\alpha\{x\}+\theta(x)$, then

$$
\alpha H(x)-\alpha H(\lfloor x\rfloor)=-\alpha^{2}\{x\}+\alpha \theta(x) .
$$

So, if $x$ is sufficiently large and not an integer then

$$
-\frac{5}{4} \alpha^{2}\{x\}<\alpha H(x)-\alpha H(\lfloor x\rfloor)<-\frac{3}{4} \alpha^{2}\{x\} .
$$

Let $n_{1}$ be the smallest integer such that any non integer $x>n_{1}$ satisfies condition (14). If $\#\left\{n \in \mathcal{S}: n \geq n_{1}\right\}=0$ then $M \leq n_{1}$, so, the lemma is clearly true for sufficiently large $T$. Otherwise,

$$
\#\left\{n \in \mathcal{S}: n \geq n_{1}\right\} \geq M-n_{1} \gg M .
$$

Take $n \in \mathcal{S}$ with $n \geq n_{1}$ and $t \in[n, n+h]$. For any integer $m \in[t, t+h]$, $\alpha H(m) \leq 0$. Now, for any $1 \leq j<h$,

$$
\int_{[t]+j}^{[t]+j+1} H(u) \mathrm{d} u=\int_{[t]+j}^{[t]+j+1}(H(u)-H([t]+j)) \mathrm{d} u+\int_{[t]+j}^{[t]+j+1} H([t]+j) \mathrm{d} u .
$$

Therefore, by (14),

$$
\begin{aligned}
\int_{[t]+j}^{[t]+j+1} \alpha H(u) \mathrm{d} u & <\int_{0}^{1}\left(-\frac{3}{4} \alpha^{2} x\right) \mathrm{d} x+\alpha H([t]+j) \\
& <-\frac{3}{8} \alpha^{2}
\end{aligned}
$$

because $\alpha H([t]+j) \leq 0$. Since $[t] \geq n$, we also have

$$
\int_{t}^{[t]+1} \alpha H(u) \mathrm{d} u<\int_{\{t\}}^{1}\left(-\frac{3}{4} \alpha^{2} x\right) \mathrm{d} x+\alpha H([t])(1-\{t\})<0
$$

and

$$
\int_{[t]+h}^{t+h} \alpha H(u) \mathrm{d} u<\int_{0}^{\{t\}}\left(-\frac{3}{4} \alpha^{2} x\right) \mathrm{d} x+\alpha H([t]+h)\{t\} \leq 0 .
$$

Hence,

$$
\left|\int_{t}^{t+h} H(u) \mathrm{d} u\right| \geq \frac{3}{8}|\alpha|(h-1)
$$


Take an integer $r=r(T)$ such that $2^{r}>(\log T)^{3+\frac{D}{2}}$. Using (6) and (9), we obtain

$$
\begin{aligned}
& \int_{0}^{2 T}\left(\int_{t}^{t+h} H(u) \mathrm{d} u\right)^{2} \mathrm{~d} t=\int_{0}^{\frac{T}{2^{r}}}\left(\int_{t}^{t+h} H(u) \mathrm{d} u\right)^{2} \mathrm{~d} t \\
&+\sum_{j=0}^{r} \int_{\frac{T}{2^{j}}}^{\frac{T}{2^{j-1}}}\left(\int_{t}^{t+h} H(u) \mathrm{d} u\right)^{2} \mathrm{~d} t \\
& \ll \frac{T}{2^{r}} h^{2}(\log T)^{2+\frac{D}{2}}+h^{\frac{3}{2}} \sum_{j=0}^{r} \frac{T}{2^{j}} \\
& \ll T h^{\frac{3}{2}}
\end{aligned}
$$

due to $h \leq \log T$. On the other hand,

$$
\begin{aligned}
\int_{0}^{2 T}\left(\int_{t}^{t+h} H(u) \mathrm{d} u\right)^{2} \mathrm{~d} t & \geq \sum_{n \in \mathcal{S}} \int_{n}^{n+h}\left(\int_{t}^{t+h} H(u) \mathrm{d} u\right)^{2} \mathrm{~d} t \\
& \geq \sum_{\substack{n \in \mathcal{S} \\
n \geq n_{1}}} \int_{n}^{n+h}\left(\frac{3}{8}|\alpha|(h-1)\right)^{2} \mathrm{~d} t \\
& \gg M h^{3} .
\end{aligned}
$$

Hence $M \leq c_{1} \frac{T}{h^{\frac{3}{2}}}$ for some absolute constant $c_{1}$.

Take $c_{0}=c / 6 h$. If $h$ is a suitably large integer such that $c_{1} T<c_{0} T h^{\frac{3}{2}}$, then there are at least $c_{0} T$ intervals $K$ in $\mathcal{D}$ such that $\alpha H(n) \prec 0$ for some integer $n \in K$ and $\alpha H(m)>0$ for some integer $m$ lying in $(n, n+2 h)$.

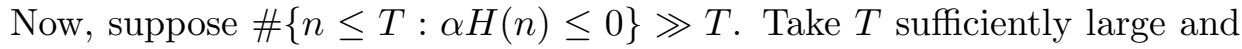
take the order relation ' $\prec$ ' to be ' $\leq$ '. Therefore, we have $c_{0} T$ integers $m$ in the interval $[1,2 T]$, for which $\alpha H(m)$ is positive. In this case,

$$
\#\{n \leq T: \alpha H(n)>0\} \gg T .
$$

If we don't have $\#\{n \leq T: \alpha H(n) \leq 0\} \gg T$, then

$$
\#\{n \leq T: \alpha H(n)>0\}=T(1+o(1)) .
$$

Hence, part 1 of Theorem 1.1 is proved. Next, we prove part 2. Take ' $\prec$ ' to be ' $<$ '. Then, there exists a positive constant $c_{0}$ and $c_{0} T$ disjoint subintervals of $[1, T]$, with each of them having at least two integers, $m$ and $n$, such that $H(m)>0$ and $H(n)<0$. Therefore, in each of those intervals we have at least one $l$ with either $H(l)=0$ or $H(l) H(l+1)<0$. Whence, $z_{H}(T)>\frac{c_{0}}{2} T$ or $N_{H}(T)>\frac{c_{0}}{2} T$. 


\section{A class of arithmetical functions}

In this section, we consider arithmetical functions $f(n)$, such that the sequence $b_{n}$ satisfies conditions (3) and (4). We begin with some elementary results about this class of arithmetical functions. Using condition (4), we immediately obtain the following lemma:

Lemma 6.1. Let $b_{n}$ be a sequence of real numbers satisfying (4), for some constants $B$ and $A>1$, then there exist constants $\gamma_{b}$ and $\alpha$ such that

$$
\begin{aligned}
& \sum_{n \leq x} \frac{b_{n}}{n}=B \log x+\gamma_{b}+O\left(\frac{1}{\log ^{A-1} x}\right), \\
& \sum_{n=1}^{\infty} \frac{b_{n}}{n^{2}}=\alpha \\
& \sum_{n>x} \frac{b_{n}}{n^{2}}=\frac{B}{x}+O\left(\frac{1}{x \log ^{A-1} x}\right) .
\end{aligned}
$$

Next, we calculate the sum of $f(n)$ and describe the error term $H(x)$.

Lemma 6.2. Let $b_{n}$ be a sequence of real numbers as in Lemma 6.1, then

$$
\sum_{n \leq x} f(n)=\sum_{n \leq x} \sum_{d \mid n} \frac{b_{d}}{d}=\alpha x-\frac{B \log 2 \pi x}{2}-\frac{\gamma_{b}}{2}+H(x),
$$

where,

$$
H(x)=-\sum_{n \leq \frac{x}{\log ^{C} x}} \frac{b_{n}}{n} \psi\left(\frac{x}{n}\right)+O\left(\frac{1}{\log ^{C} x}\right)+O\left(\frac{1}{\log ^{A-C-1} x}\right),
$$

for any $0<C<A-1$.

Proof. We have

$$
\sum_{n \leq x} f(n)=\sum_{n \leq x} \sum_{d \mid n} \frac{b_{d}}{d}=\sum_{d \leq x} \frac{b_{d}}{d} \sum_{\substack{n \leq x \\ d \mid n}} 1=\sum_{m \leq x} \sum_{d \leq \frac{x}{m}} \frac{b_{d}}{d} .
$$

We separate the double sum above in two parts. Let $0<C<A-1$ and $y=\log ^{C} x$. Then

$$
\sum_{m \leq x} \sum_{d \leq \frac{x}{m}} \frac{b_{d}}{d}=\sum_{n \leq y} \sum_{d \leq \frac{x}{n}} \frac{b_{d}}{d}+\sum_{d \leq \frac{x}{y}} \frac{b_{d}}{d} \sum_{y<n \leq \frac{x}{d}} 1 .
$$


In order to evaluate the first term on the right, we start with an application of formula (15):

$$
\begin{aligned}
\sum_{n \leq y} \sum_{d \leq \frac{x}{n}} \frac{b_{d}}{d} & =\sum_{n \leq\lfloor y\rfloor}\left(B \log x-B \log n+\gamma_{b}+O\left(\frac{1}{\log ^{A-1}\left(\frac{x}{n}\right)}\right)\right) \\
& =B\lfloor y\rfloor \log x-B \sum_{n \leq\lfloor y\rfloor} \log n+\gamma_{b}\lfloor y\rfloor+O\left(\frac{y}{\log ^{A-1}\left(\frac{x}{y}\right)}\right) .
\end{aligned}
$$

Recall that by Stirling formula, $\sum_{n \leq\lfloor y\rfloor} \log n=\lfloor y\rfloor \log \lfloor y\rfloor-\lfloor y\rfloor+\frac{\log \lfloor y\rfloor}{2}+$ $\frac{\log 2 \pi}{2}+O\left(\frac{1}{y}\right)$. Hence,

$$
\begin{aligned}
\sum_{n \leq y} \sum_{d \leq \frac{x}{n}} \frac{b_{d}}{d}=B\lfloor y\rfloor(\log x-\log \lfloor y\rfloor+1)+ & \gamma_{b}\lfloor y\rfloor-\frac{B(\log 2 \pi y)}{2} \\
& +O\left(\frac{y}{\log ^{A-1} x}\right)+O\left(\frac{1}{y}\right) .
\end{aligned}
$$

For the second term, we get

$$
\begin{aligned}
\sum_{d \leq \frac{x}{y}} \frac{b_{d}}{d} \sum_{y<n \leq \frac{x}{d}} 1 & =\sum_{d \leq \frac{x}{y}} \frac{b_{d}}{d}\left(\left\lfloor\frac{x}{d}\right\rfloor-\lfloor y\rfloor\right) \\
= & x \sum_{d=1}^{\infty} \frac{b_{d}}{d^{2}}-x \sum_{d>\frac{x}{y}} \frac{b_{d}}{d^{2}}-\sum_{d \leq \frac{x}{y}} \frac{b_{d}}{d} \psi\left(\frac{x}{d}\right)-\left(\frac{1}{2}+\lfloor y\rfloor\right) \sum_{d \leq \frac{x}{y}} \frac{b_{d}}{d} \\
= & \alpha x-B y-\sum_{d \leq \frac{x}{y}} \frac{b_{d}}{d} \psi\left(\frac{x}{d}\right)-\frac{\gamma_{b}+B \log x}{2} \\
& -B\lfloor y\rfloor(\log x-\log y)+\frac{B \log y}{2}-\gamma_{b}\lfloor y\rfloor+O\left(\frac{y}{\log ^{A-1} x}\right) .
\end{aligned}
$$

Notice also that $B\lfloor y\rfloor(\log y-\log \lfloor y\rfloor)=B\lfloor y\rfloor\left(-\log \left(1-\frac{\{y\}}{y}\right)\right)=B\{y\}+$ $O\left(\frac{1}{y}\right)$. Joining everything together, we obtain

$$
\begin{aligned}
H(x) & =\sum_{n \leq x} f(n)-\left(\alpha x-\frac{B \log 2 \pi x}{2}-\frac{\gamma_{b}}{2}\right) \\
& =-\sum_{d \leq \frac{x}{\log ^{C} x}} \frac{b_{d}}{d} \psi\left(\frac{x}{d}\right)+O\left(\frac{1}{\log ^{A-C-1} x}\right)+O\left(\frac{1}{\log ^{C} x}\right) .
\end{aligned}
$$


Proof of Theorem 1.2: We just have to show that $H(x)$ satisfies the conditions of Theorem 1.1. From Lemma 6.2, for any $x$

$$
\begin{aligned}
H(x)-H(\lfloor x\rfloor) & =-\alpha\{x\}-\frac{B}{2}(\log 2 \pi\lfloor x\rfloor-\log 2 \pi x) \\
& =-\alpha\{x\}+\frac{B}{2} \frac{\{x\}}{x}+O\left(\frac{1}{x^{2}}\right) .
\end{aligned}
$$

In Lemma 6.2, we also obtained

$$
H(x)=-\sum_{n \leq \frac{x}{\log ^{C} x}} \frac{b_{n}}{n} \psi\left(\frac{x}{n}\right)+O\left(\frac{1}{\log ^{C} x}\right)+O\left(\frac{1}{\log ^{A-C-1} x}\right),
$$

for any $0<C<A-1$. Take $C=5+\frac{D}{2}, y(x)=\frac{x}{\log ^{C} x}$ and $k(x)=$ $\min \left(\log ^{C} x, \log ^{A-C-1} x\right)$. Since $A>6+D / 2$, then $C<A-1$ and $A-C-1>0$. The first part of Theorem 1.2 now follows from Theorem 1.1.

Suppose that $f(n)$ takes only rational values. In order to prove the second part of Theorem 1.2, we use the following result of A. Baker [1].

Proposition. Let $\alpha_{1}, \ldots, \alpha_{n}$ and $\beta_{0}, \ldots, \beta_{n}$ denote nonzero algebraic numbers. Then $\beta_{0}+\beta_{1} \log \alpha_{1}+\cdots+\beta_{n} \log \alpha_{n} \neq 0$.

Using the result above, we obtain the next lemma.

Lemma 6.3. Let $f(n)$ be a rational valued arithmetical function and suppose the sequence $b_{n}$ satisfies condition (4) for some real $B$ and $A>1$. Let $r$ be a real number and suppose $H(x)$ is given by (18). Then

(1) If $B=0$ and $\alpha$ is irrational then $\#\{n$ integer: $H(n)=r\} \leq 1$;

(2) If $B$ is a nonzero algebraic number then \# $\{n$ integer: $H(n)=r\} \leq 2$;

(3) If $B$ is transcendental then there exists a constant $C$ that depends on $r$ and on the function $f(n)$, such that

$$
\#\{n \leq T, n \text { integer }: H(n)=r\}<(\log T)^{C} .
$$

Proof. Suppose that $B=0$ and $\alpha$ is irrational. Suppose also that there are two integers, say $M \neq N$, such that $H(M)=H(N)$. Then

$$
\sum_{n \leq M} f(n)-\alpha M+\frac{\gamma_{b}}{2}=\sum_{n \leq N} f(n)-\alpha N+\frac{\gamma_{b}}{2} .
$$

But this implies that $\alpha$ is rational, a contradiction.

Next, suppose $B \neq 0$ is algebraic number and that there are $M>N>Q$ integers, satisfying $H(M)=H(N)=H(Q)$. We have

$$
\sum_{n \leq M} f(n)-\alpha M+\frac{B \log 2 \pi M}{2}+\frac{\gamma_{b}}{2}=\sum_{n \leq N} f(n)-\alpha N+\frac{B \log 2 \pi N}{2}+\frac{\gamma_{b}}{2}
$$


which implies

$$
\alpha=\frac{B}{M-N} \log \left(\frac{M}{N}\right)+\frac{1}{M-N} \sum_{N<n \leq M} f(n) .
$$

Consequently

$$
B \log \left(\frac{\left(\frac{M}{N}\right)^{\frac{1}{M-N}}}{\left(\frac{M}{Q}\right)^{\frac{1}{M-Q}}}\right)=\frac{1}{M-Q} \sum_{Q<n \leq M} f(n)-\frac{1}{M-N} \sum_{N<n \leq M} f(n) .
$$

We are going to prove that

$$
\left(\frac{M}{N}\right)^{\frac{1}{M-N}} \neq\left(\frac{M}{Q}\right)^{\frac{1}{M-Q}} .
$$

Since $B$ is a nonzero algebraic number and the values of $f(n)$ are rational, for any integer $n$, the proposition implies

$$
B \log \left(\frac{\left(\frac{M}{N}\right)^{\frac{1}{M-N}}}{\left(\frac{M}{Q}\right)^{\frac{1}{M-Q}}}\right) \neq \frac{1}{M-Q} \sum_{Q<n \leq M} f(n)-\frac{1}{M-N} \sum_{N<n \leq M} f(n),
$$

and so we get a contradiction, which implies $\#\{n$ integer : $H(n)=r\} \leq 2$, for any real number $r$. In fact, instead of proving (21), we are going to prove that

$$
M^{N-Q} Q^{M-N}<N^{M-Q},
$$

for any positive integers $M>N>Q$. Clearly, this implies (21). The inequality (22) is just a particular case of the geometric mean-analytic mean inequality

$$
\left(\prod_{i=1}^{n} u_{i}\right)^{\frac{1}{n}} \leq \frac{1}{n} \sum_{i=1}^{n} u_{i}
$$

where equality happens only if $u_{1}=u_{2}=\cdots=u_{n}$. In fact, if we take $n=M-Q, u_{i}=M$ for $1 \leq i \leq N-Q$ and $u_{i}=Q$ for $N-Q<i \leq M-Q$, we derive

$$
\left(M^{N-Q} Q^{M-N}\right)^{\frac{1}{M-Q}}<\frac{1}{M-Q}((N-Q) M+(M-N) Q)=N .
$$

Hence, we obtain (22) and part 2 of the Lemma.

Finally we prove part 3 . Suppose $r$ is a real number such that

$$
\#\{n \leq T: H(n)=r\} \geq 4 .
$$


Let $Q<N<M$ be the three smallest positive integers in the above set, then

$$
0 \neq B \log \left(\frac{\left(\frac{M}{N}\right)^{\frac{1}{M-N}}}{\left(\frac{M}{Q}\right)^{\frac{1}{M-Q}}}\right)=\frac{1}{M-Q} \sum_{Q<n \leq M} f(n)-\frac{1}{M-N} \sum_{N<n \leq M} f(n) .
$$

Suppose $L$ is such that $H(L)=r$. Then $L>N>Q$, and as in part 2:

$$
0 \neq B \log \left(\frac{\left(\frac{L}{N}\right)^{\frac{1}{L-N}}}{\left(\frac{L}{Q}\right)^{\frac{1}{L-Q}}}\right)=\frac{1}{L-Q} \sum_{Q<n \leq L} f(n)-\frac{1}{L-N} \sum_{N<n \leq L} f(n) .
$$

After we cross multiply the two expressions above, we obtain

$$
\log \left(\frac{\left(\frac{M}{N}\right)^{\frac{1}{M-N}}}{\left(\frac{M}{Q}\right)^{\frac{1}{M-Q}}}\right)=r_{1} \log \left(\frac{\left(\frac{L}{N}\right)^{\frac{1}{L-N}}}{\left(\frac{L}{Q}\right)^{\frac{1}{L-Q}}}\right)
$$

for some rational $r_{1}$. Therefore, there are four rational numbers $r_{2}, r_{3}, r_{4}$ and $r_{5}$, such that

$$
L^{r_{2}}=M^{r_{3}} N^{r_{4}} Q^{r_{5}} .
$$

Now, any prime dividing $L$ must divide $M N Q$. Notice that, if $p$ is a prime, $k$ is an integer and $p^{k} \leq x$ then $k \leq \frac{\log x}{\log p}$. Therefore, the number of integers smaller than $x$, which have all prime divisors smaller than $M$ is smaller than $(\log x)^{\pi(M)}$. This finishes the proof.

Except when $\alpha=0$, or $B=0$ and $\alpha$ is rational, we cannot have $z_{H}(T) \gg$ $T$. Hence, we obtain the second part of Theorem 1.2.

Example. We finish this section by proving that Theorem 1.2 is valid for the arithmetical function $\frac{n}{\phi(n)}$.

Notice that

$$
\frac{n}{\phi(n)}=\prod_{p \mid n}\left(1+\frac{1}{p-1}\right)=\sum_{d \mid n} \frac{\mu^{2}(d)}{\phi(d)} .
$$

Let $b_{n}=\frac{\mu^{2}(n) n}{\phi(n)}$, then $f(n)=\sum_{d \mid n} \frac{b_{d}}{d}$. In [8], R. Sitaramachandrarao proved that

$$
\sum_{n \leq x} \frac{\mu^{2}(n) n}{\phi(n)}=x+O\left(x^{\frac{1}{2}}\right)
$$


so condition (4) is satisfied for any $A$ and with $B=1$. By Merten's Theorem $\prod_{p \mid n}\left(1-\frac{1}{p}\right)^{-1} \leq \prod_{p \leq n}\left(1-\frac{1}{p}\right)^{-1} \sim e^{\gamma} \log n$, hence

$$
\sum_{n \leq x} b_{n}^{4}=\sum_{n \leq x} \mu^{2}(n) \frac{n^{4}}{\phi^{4}(n)}=\sum_{n \leq x} O\left(\log ^{4} n\right)=O\left(x \log ^{4} x\right),
$$

and condition (3) is satisfied for $D \geq 4$. In this case,

$$
\alpha=\frac{\zeta(2) \zeta(3)}{\zeta(6)}, \quad \gamma_{b}=\gamma+\sum_{p} \frac{\log p}{p(p-1)}
$$

and

$$
H(x)=\sum_{n \leq x} \frac{n}{\phi(n)}-\frac{\zeta(2) \zeta(3)}{\zeta(6)} x+\frac{\log x}{2}+\frac{\log 2 \pi+\gamma+\sum_{p} \frac{\log p}{p(p-1)}}{2} .
$$

Since $B=1$ we can apply Theorem 6.3 , and so $z(T) \leq 2$. Therefore, if $\#\{n \leq T: \alpha H(n)<0\} \gg T$, then $N_{H}(T) \gg T$.

\section{Second class of arithmetical functions}

Given a sequence of real numbers $b_{n}$, and a complex number $s$, we define the Dirichlet series $B(s)=\sum_{n=1}^{\infty} \frac{b_{n}}{n^{s}}$. In this section, we consider arithmetical functions $f(n)$, such that the sequence $b_{n}$ satisfies conditions (3) and (5) for some $D>0, \beta$ real and a function $g(s)$ with a Dirichlet series expansion absolutely convergent for $\sigma>1-\lambda$, for some $\lambda>0$.

U. Balakrishnan and Y.-F. S. Pétermann [2] proved that:

Proposition. Let $f(n)$ be a complex valued arithmetical function satisfying

$$
\sum_{n=1}^{\infty} \frac{f(n)}{n^{s}}=\zeta(s) \zeta^{\beta}(s+1) g(s+1),
$$

for a complex number $\beta$, and $g(s)$ having a Dirichlet series expansion

$$
g(s)=\sum_{n=1}^{\infty} \frac{c_{n}}{n^{s}}
$$

which is absolutely convergent in the half plane $\sigma>1-\lambda$ for some $\lambda>0$. Let $\beta_{0}$ be the real part of $\beta$. If

$$
\zeta^{\beta}(s) g(s)=\sum_{n=1}^{\infty} \frac{b_{n}}{n^{s}}
$$


then there is a real number $b, 0<b<1 / 2$, and constants $B_{j}$, such that, taking $y(x)=x \exp \left(-(\log x)^{b}\right)$ and $\alpha=\zeta^{\beta}(2) g(2)$,

$\sum_{n \leq x} f(n)= \begin{cases}\alpha x-\sum_{n \leq y(x)} \frac{b_{n}}{n} \psi\left(\frac{x}{n}\right)+o(1) & \text { if } \beta_{0}<0, \\ \alpha x+\sum_{j=0}^{\left[\beta_{0}\right]} B_{j}(\log x)^{\beta-j}-\sum_{n \leq y(x)} \frac{b_{n}}{n} \psi\left(\frac{x}{n}\right)+o(1) & \text { if } \beta_{0} \geq 0,\end{cases}$

The real version of the previous proposition allows us to prove Theorem 1.3:

Proof. Notice that, for any $c>0, \log ^{c}\lfloor x\rfloor=\log ^{c} x-c \frac{\{x\}}{x} \log ^{c-1} x+O\left(\frac{1}{x}\right)$. So, $H(x)=H(\lfloor x\rfloor)-\alpha\{x\}+o(1)$. From the previous proposition, there is an increasing function $k(x)$, with $\lim _{x \rightarrow \infty} k(x)=\infty$, such that

$$
H(x)=-\sum_{n \leq y(x)} \frac{b_{n}}{n} \psi\left(\frac{x}{n}\right)+O\left(\frac{1}{k(x)}\right),
$$

where $y(x)=x \exp \left(-(\log x)^{b}\right)$, for some $0<b<1 / 2$. Hence, the result follows from Theorem 1.1.

Acknowledgements: I would like to thank my advisor Andrew Granville for his guidance and encouragement in this research and to the Referee for his useful comments.

\section{References}

[1] A. BAKER, Linear forms in the logarithms of algebraic numbers (III). Mathematika 14 (1967), 220-228.

[2] U. Balakrishnan, Y.-F. S. Pétermann, The Dirichlet series of $\zeta(s) \zeta^{\alpha}(s+1) f(s+1)$ : On an error term associated with its coefficients. Acta Arithmetica 75 (1996), 39-69.

[3] S. CHowlA, Contributions to the analytic theory of numbers. Math. Zeit. 35 (1932), 279299.

[4] G. H. Hardy, E. M. Wright, An Introduction to the Theory of Numbers. 5th ed. Oxford 1979.

[5] Y.-K. LAU, Sign changes of error terms related to the Euler function. Mathematika 46 (1999), 391-395.

[6] Y.-F. S. PÉtermann, On the distribution of values of an error term related to the Euler function. Théorie des Nombres, (Quebec, PQ, 1987), 785-797.

[7] S. Ramanujan, Collected papers. Cambridge, 1927, 133-135.

[8] R. Sitaramachandrarao, On an error term of Landau - II. Rocky Mount. J. of Math. 152 (1985), 579-588.

[9] A. Walfisz, Teilerprobleme II. Math. Zeit. 34 (1931), 448-472.

Paulo J. AlmeidA

Departamento de Matemática

Universidade de Aveiro

Campus Universitário de Santiago

3810-193 Aveiro, Portugal

E-mail : paulo@mat.ua.pt 